\title{
Effects of Uric Acid on Hearts of Rats with Chronic Kidney Disease
}

\author{
Xiaorong $\mathrm{Bao}^{\mathrm{a}}$ Yu Wang $^{\mathrm{a}}$ Cuifang Wei $^{\mathrm{b}}$ Qing Zhang ${ }^{\mathrm{a}}$ \\ ${ }^{a}$ Department of Nephrology, Jinshan Hospital affiliated to Fudan University, Jinshan District, Shanghai and \\ ${ }^{b}$ Department of Nephrology, Suzhou Municipal Hospital, Suzhou, Jiangsu Province, China
}

\author{
Key Words \\ CKD · Early stage $\cdot$ UA · Cardiac lesion · Allopurinol · \\ Micro-inflammation
}

\begin{abstract}
Aims: To study the effects of uric acid on cardiac lesions and its possible mechanism by establishing an early-stage CKD animal model with hyperuricemia. Allopurinol was used to see whether it could reduce cardiac lesions. Methods: The experimental rats were randomly divided into 4 groups $(\mathrm{n}=$ 15): Group A (sham-operative group), Group B (CKD group), Group C (CKD with hyperuricemia group), Group D (CKD with hyperuricemia + allopurinol group). After 16 weeks, the rats were sacrificed and blood samples were collected for detection of Scr, SUA, hs-CRP and IL-6. Kidney and heart tissues were pathologically examined. The collagen I of heart tissues was examined by immunohistochemistrical methods. $\boldsymbol{R} \boldsymbol{e}$ sults: Obvious pathological changes could be observed in Group C. However, compared to Group C, the pathological changes in Group D were lighter. The proportion of collagen I positive area (PCIPA) in Group $C$ was significantly higher than that in Group A, B and D. Univariate analysis showed that the SUA level had a significant positive correlation with PCIPA in myocardium. IL- 6 and hs-CRP levels in Group C were significantly higher than in Group A, B and D. Univariate analysis showed that the SUA level had a significant positive correlation with IL- 6 and hs-CRP, and PCIPA in myocardium had a significant positive correlation with hs-CRP and IL-6 levels.
\end{abstract}

Conclusions: There were obvious cardiac lesions in earlystage CKD rats with hyperuricemia, and the severity of cardiac lesions was positively related to the level of SUA. Microinflammation might be one mechanism causing cardiac lesions. Allopurinol could alleviate cardiaclesions in early-stage CKD rats by lowering the SUA level, which, in turn, could reduce the severity of micro-inflammation.

(c) 2014 S. Karger AG, Basel

Since previous studies have highlighted the role of serum uric acid (UA) in coronary heart disease [1], the effect and mechanism of UA in cardiovascular disease (CVD) have aroused widespread concern. Researches [2, 3] have found that uric acid could stimulate vascular smooth muscle cell proliferation in rats, and also induce endothelial dysfunction. The serum UA level is not only a significant predictor of occurrence of primary hypertension [4]; it is also associated with cardiovascular morbidity and mortality [5-7]. Chicago Heart Study, NHANES III study, SHEP research all drew the conclusion that UA was connected to CVD occurrence through large-scale epidemiological survey. All these researches based on the general population or primary hypertension patients indicate the important role of UA in the occurrence of CVD.

The incidence of chronic kidney disease (CKD) is 10$13 \%$. CKD patients have an extremely higher risk of CVD than the general population. CVD is the major cause of

\begin{tabular}{ll}
\hline KARGER & $\begin{array}{l}\text { ( ) 2014 S. Karger AG, Basel } \\
0250-8095 / 14 / 0404-0308 \$ 39.50 / 0 \quad \text { Karger }\end{array}$ \\
$\begin{array}{l}\text { E-Mail karger@karger.com } \\
\text { www.karger.com/ajn }\end{array}$ & $\begin{array}{l}\text { This is an Open Access article licensed under the terms of the } \\
\text { Creative Commons Attribution-NonCommercial 3.0 Un- } \\
\text { ported license (CC BY-NC) (www.karger.com/OA-license), } \\
\text { applicable to the online version of the article only. Distribu- } \\
\text { tion permitted for non-commercial purposes only. }\end{array}$
\end{tabular}


death in patients with CKD [8]. The increase in serum UA occurs in the early and middle stages of CKD and happens even more with the deterioration of the kidney function [9]. The relation between UA and CVD has been confirmed in the general population; however, the role and mechanism of UA in the occurrence of CVD in early-stage CKD are still unknown. Our earlier study [10] found that the level of SUA had a substantial positive correlation with endothelial dysfunction (ED) in early CKD rats, indicating that hyperuricemia was associated with CVD in early CKD.

Allopurinol is a competitive inhibitor of xanthine oxidase, which could reduce the generation of UA. As a traditional SUA-lowering drug, allopurinol is routinely used to treat gout. Recent studies $[11,12]$ have discovered the cardiovascular protection function of allopurinol by inhibiting oxidative stress and inflammatory responses.

Therefore, by establishing an early-stage CKD animal model with hyperuricemia, we endeavored to study the relationship between UA and cardiac lesions in early-stage $\mathrm{CKD}$ and its possible mechanism. Meanwhile, we tried to intervene the process with allopurinol to observe its effects on cardiac lesions to provide the basis for clinical medication.

\section{Methods}

\section{The Establishment of Early CKD Animal Model with}

\section{Hyperuricemia}

60 male Sprague-Dawley rats, weighing 232 to $271 \mathrm{~g}$ and 6-7 weeks old, obtained from the animal laboratory of Shanghai Medical College, Fudan University (Shanghai, China), were employed in this research. All experimental procedures were conducted in accordance with the Guiding Principles for the Care and Use of Animals in Research and Teaching, approved by the Institutional Animal Care and Use Committee of Jinshan Hospital affiliated to Fudan University, China.

The experimental animals were randomly divided into 4 groups as follows: Group A (sham-operative group, $\mathrm{n}=15$ ) served as the normal control, Group B (CKD group, $\mathrm{n}=15)$, in which rats only received right nephrectomy, Group $\mathrm{C}$ (CKD with hyperuricemia group, $n=15)$, in which rats were right nephrectomized and then gavaged with potassium oxonate (OXO) $(800 \mathrm{mg} / \mathrm{kg}$, twice a day), Group D (CKD with hyperuricemia + allopurinol group, $n=15)$, in which rats were right nephrectomized, and then gavaged with $\mathrm{OXO}$ (as in Group C) and allopurinol ( $25 \mathrm{mg} / \mathrm{kg}$, twice a day). The rats were housed in standard plastic cages; food and water were freely available.

Rats were anesthetized with an intraperitoneal injection of $5 \%$ ketamine $(100 \mathrm{mg} / \mathrm{kg})$. The surgical region was shaved and cleaned with $75 \%$ alcohol. The right kidney was exposed through a longitudinal incision under the right costal arch and (proximal to the right side of the spine). After the separation of perirenal fat and renal capsule, renal pedicle was clamped and ligated. The right kidney was then resected with scissors. Layers of tissue and skin were then sutured if no bleeding was observed after the release of vascular clips. Chlortetracycline was then applied to the incision. The entire procedure mentioned above was performed in Group A, but nephrectomy was not applied. After one week of normal feeding, the rats in all four groups were in good condition. Rats in Group $\mathrm{C}$ and $\mathrm{D}$ were fed with drugs by gavage. The dose of OXO was $800 \mathrm{mg} / \mathrm{kg}$ twice a day, and allopurinol $25 \mathrm{mg} / \mathrm{kg}$ twice a day. Rats in Group A and B were gavaged with saline with the same amount as in Group C and D. During the experiment, rats were weighed every two weeks and the administered dose was adjusted based on body weight.

\section{Reagents and Instruments}

hs-CRP and IL- 6 detection kits were purchased from Nanjing KeyGEN Biotech. Co. Ltd. (Nanjing, China). DAB chromogenic kit, PBS buffer (powdered) and citrate buffer (powdered) were purchased from Fuzhou Maixin Biotechnology Co. Ltd. (Fujian, China). Rabbit anti-mouse collagen I polyclonal antibody and goat anti-rabbit polyclonal antibody were purchased from Boster Biotechnology Co. Ltd. (Wuhan, China). Beckman CX9 biochemical analyzer and Beckman supporting reagents were purchased from Beckman Coulter Inc. (USA). $-70^{\circ} \mathrm{C}$ refrigerator was purchased from SANYO Electric Co. Ltd. (Japan).

\section{Sample Collection and Management}

After 16 weeks of gavage administration, rats were sacrificed and blood samples were collected from the heart into non-heparinized tubes. The blood sera was then collected via centrifugation and stored at $-70^{\circ} \mathrm{C}$ for detection of UA, Scr, hs-CRP and IL-6. The heart was then obtained by cutting the roots of the pulmonary artery, pulmonary vein, aorta and vena cava. A part of anterior wall of the left ventricle was cut and fixed with $10 \%$ formalin for at least $8 \mathrm{~h}$, and then embedded with paraffin. After routine processing, the paraffin sections of each tissue were cut into slices for hematoxylin-eosin (HE) staining for light microscopic examination and determination of collagen I by immunohistochemistrical methods. Meanwhile, a longitudinal incision was made to obtain the tissues of the left kidney, which were fixed with $10 \%$ formalin and stored at $4^{\circ} \mathrm{C}$ for 14 to $16 \mathrm{~h}$. They were then placed in $70 \%$ alcohol at $4^{\circ} \mathrm{C}$ refrigerator for HE staining. Observation of slices was done by pathologists who were not participating in this experiment.

\section{The Detection of Serologic Indexes}

The detection of Scr and SUA was performed according to the instructions by the kits. IL- 6 and hs-CRP were assayed using commercial enzyme-linked immunosorbent assay (ELISA) kits.

\section{Statistical Analysis}

The proportion of collagen I positive area (PCIPA) was calculated by randomly selecting 10 fields in each slide and dividing each one into 1,564 parts using Photoshop. The number of positive points $(\mathrm{N})$ was counted by two pathologists not participating in the experiment. PCIPA was N/1, 564. Data was calculated by two examiners and the average values were calculated.

All statistical analysis was performed using the Statistical Package for Social Sciences (SPSS, Version 11.5). All data were expressed by $\overline{\mathrm{x}} \pm \mathrm{s}$. Comparisons between groups were analyzed using the $t$ test. Differences in the various parameters were assessed using oneway analysis of variance (ANOVA). A p value $<0.05$ was considered significant. 

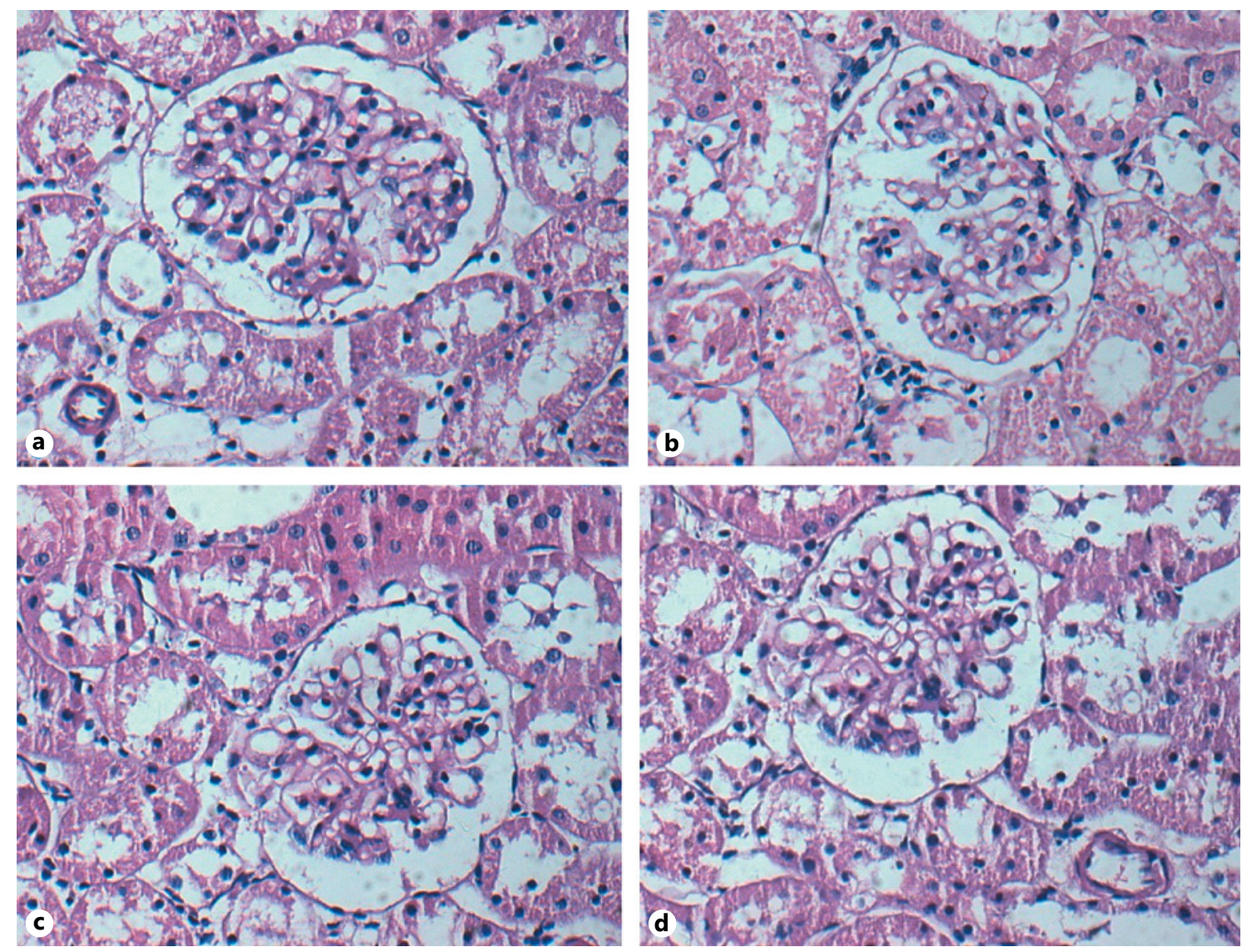

Fig. 1. a Group A kidney: normal renal glomerulus and tubules. b Group B kidney. c Group C kidney. d Group D kidney. b-d Mild glomerular mesangial proliferation and normal tubules. $257 \times 199 \mathrm{~mm}(300 \times 300 \mathrm{DPI})$. a-d HE. $\times 200$.

Table 1. Concentrations of Scr, UA, in 4 groups $(\overline{\mathrm{x}} \pm s)$

\begin{tabular}{lcc}
\hline Group & Scr, $\mu \mathrm{mol} / 1$ & $\mathrm{UA}, \mu \mathrm{mol} / 1$ \\
\hline A & $38.40 \pm 3.00$ & $73.60 \pm 9.16$ \\
B & $40.20 \pm 3.49$ & $80.93 \pm 10.55$ \\
C & $41.53 \pm 3.80$ & $125.80 \pm 15.34^{\triangle, *}$ \\
D & $41.67 \pm 3.90$ & $77.60 \pm 8.73^{\triangle}$ \\
\hline
\end{tabular}

${ }^{\triangle} \mathrm{p}<0.01$ vs. group A. ${ }^{*} \mathrm{p}<0.01$ vs. group B.

$\# \mathrm{p}<0.01$ vs. group C.

\section{Results}

Early CKD Animal Model with Hyperuricemia

No rats in any group died during the experiment. No pathological changes in glomerulus, renal tubules or renal interstitium were observed in Group A (fig. 1a). In Groups B, C and D, mild glomerular mesangial proliferation was observed with no renal tubular epithelial cell necrosis, inflammatory cells in the interstitium or small ves- sel lesions (fig. 1b-d). No urate crystal deposition was observed in any group. Compared with Groups A, B and D, the level of UA was significantly higher in Group C ( $p<$ 0.01 ). However, there was no obvious difference in the $\mathrm{Scr}$ level among the 4 groups (table 1). All these results indicate that we established an early CKD animal model with hyperuricemia successfully.

\section{Cardiac Lesions}

In the light microscope, myocardial cells in Group A were evenly arranged and fusiform, without infiltration of inflammatory cells, congestion or edema in the cardiac interstitium or the appearance of fibroblasts in vascular walls (fig. 2a, b). A small number of scattered inflammatory cells and occasional appearance of fibroblasts in large vessel walls were observed in Group B (fig. 2c, d). Myocardial cells in Group C were arranged in order with an almost uniform morphology, with obvious accumulation and infiltration of inflammatory cells, congestion in the cardiac interstitium and obvious fibrillation of small blood vessels, 

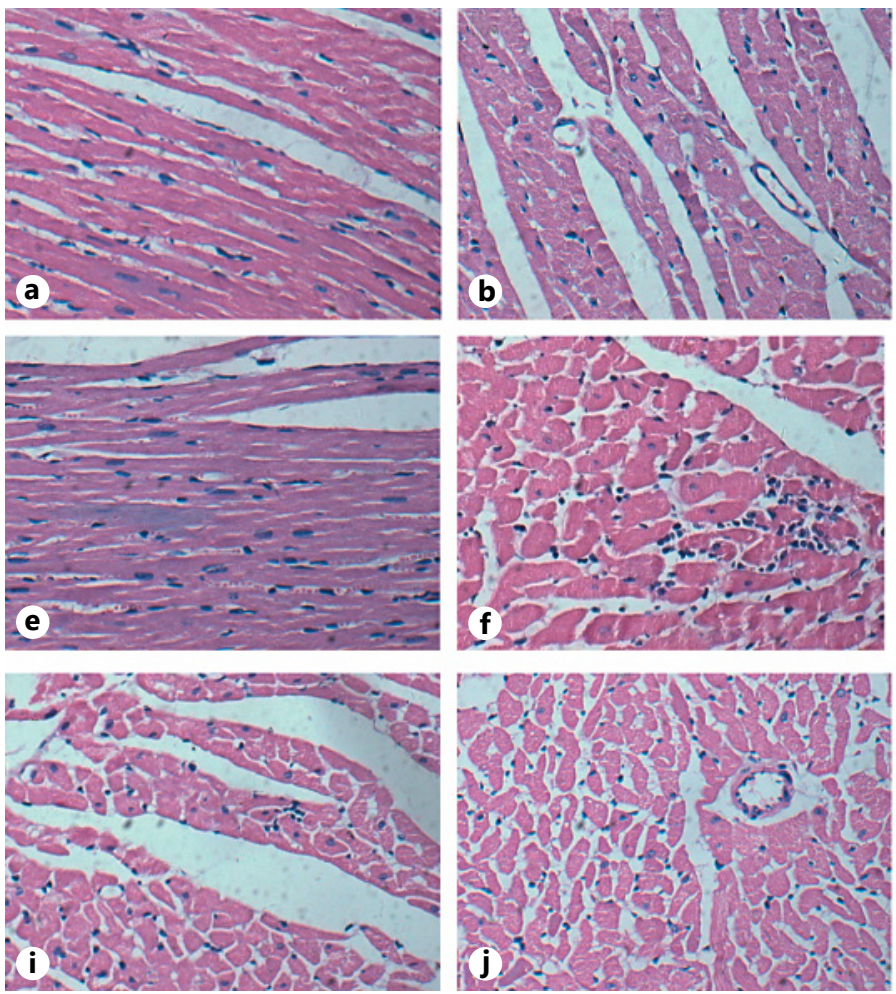

Fig. 2. a, b Group A heart: myocardial cells were evenly arranged, without inflammatory cells, congestion, edema or fibroblasts. c Group B heart: myocardial cells were evenly arranged, with few inflammatory cells, no congestion, or fibroblasts. d Group B heart: filaments around large vessel wall, without fibroblasts or degeneration. e Group C heart: myocardial cells were evenly arranged, with congestion in the interstitium. f Group $C$ heart: obvious in-

with large numbers of fibroblasts and inflammatory cells around. Hyaline degeneration was observed in some of the vessel walls (fig. $2 \mathrm{e}-\mathrm{h}$ ). Only a small number of scattering inflammatory cells with occasional fibroblasts in large vessel walls were observed in Group D (fig. 2i, j).

Stainings of collagen $I$ in four groups are shown in figure 3. No obvious collagen I deposition was visible in Group A. A small number of collagen I deposition was observed in Group B. There was significantly more amount of collagen I in Group C than that in Group B, while collagen I deposition in Group D was significantly less than that in Group C. PCIPA in Group C was $34.10 \pm 4.18 \%$, which was significantly higher than in Groups A $(24.60 \pm$ $3.82 \%), \mathrm{B}(26.38 \pm 4.09 \%)$ and $\mathrm{D}(26.55 \pm 3.92 \%)(\mathrm{p}<0.01)$.

\section{The Correlation between UA and Cardiac Lesions and its Mechanism}

The level of UA had a significant positive correlation with PCIPA (table 3), which indicated that UA was re-
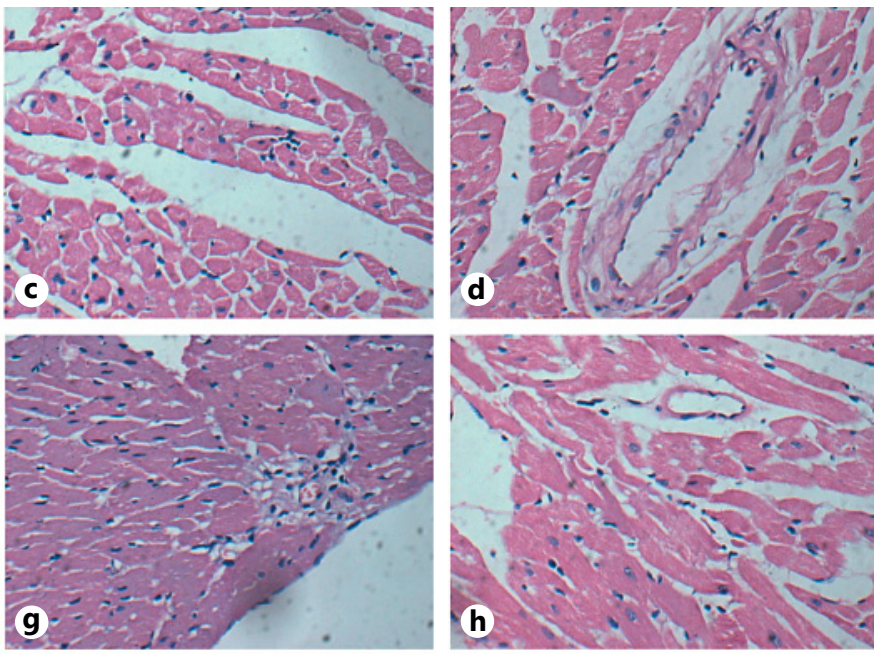

filtration of inflammatory cells. g Group C heart: obvious fibrillation. $\mathbf{h}$ Group C heart: fibroblasts in small vessel walls without degeneration. i Group D heart: slight infiltration of inflammatory cells with no interstitial fibrillation or congestion. $\mathbf{j}$ Group D heart: normal vessel walls with few filaments and no fibroblasts or degeneration. $96 \times 198 \mathrm{~mm}(300 \times 300$ DPI $) . \mathbf{a}-\mathbf{j}$ HE. $\times 200$.

lated to the increase of collagen I deposition. The levels of sera hs-CRP and IL-6 in Group C were significantly higher than in Groups A, B and D (table 2). The level of SUA had a significant positive correlation with hs-CRP and IL-6 level (table 4), which indicated that hyperuricemia could induce micro-inflammation in early CKD, and the severity of micro-inflammation was related to the UA level. The severity of micro-inflammation could be reduced by allopurinol by reducing the UA level. PCIPA was in substantial positive correlation with hs-CRP and IL-6 level (table 5), which indicated that hyperuricemia might induce cardiac lesions by micro-inflammation.

\section{Discussion}

In this research, we established an early-stage CKD animal model with hyperuricemia by right nephrectomy and gavaging the animals with OXO. There were only slight 

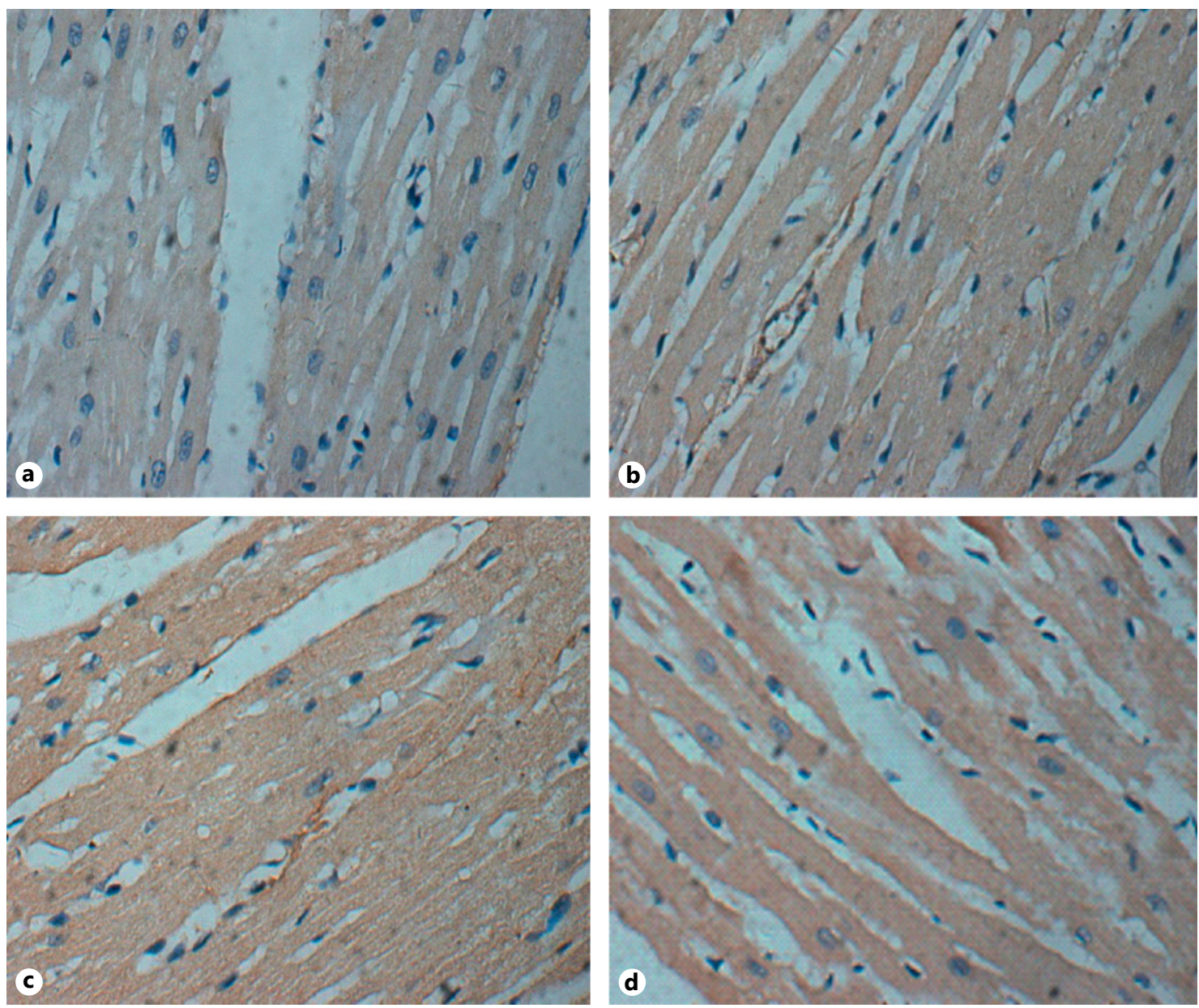

Fig. 3. a Group A heart: scarce distribution of collagen I in myocardium. b Group B heart: slight increase of collagen I in myocardium. c Group C heart: obvious increase of collagen I in myocardium. d Group D heart: slight increase of collagen I in myocardium. $220 \times 199 \mathrm{~mm}(300 \times 300 \mathrm{DPI})$. a-d Immunohistochemistry. $\times 200$.

Table 2. Concentrations of IL- 6 and hs-CRP in 4 groups $(\overline{\mathrm{x}} \pm s)$

\begin{tabular}{lll}
\hline Group & hs-CRP, ng/ml & IL-6, pg/ml \\
\hline A & $268.05 \pm 9.86$ & $26.62 \pm 0.79$ \\
B & $274.06 \pm 11.82$ & $27.58 \pm 1.27$ \\
C & $286.59 \pm 7.52^{\triangle, *}$ & $29.51 \pm 2.35^{\triangle,}$ ム \\
D & $276.10 \pm 6.30^{* *}$ & $27.72 \pm 1.44^{* *}$ \\
\hline
\end{tabular}

$\triangle \mathrm{p}<0.01$ vs. group A. ${ }^{*} \mathrm{p}<0.01$ vs. group B.

$\Delta \mathrm{p}<0.05$ vs. group B. ${ }^{* *} \mathrm{p}<0.05$ vs. group C.

pathological changes with occasional glomerular mesangial proliferation and no obvious tubal or interstitial abnormality in the kidney tissues. SUA level increased significantly, but there was no obvious difference in Scr levels among the 4 groups. Therefore, we established an earlystage CKD animal model with hyperuricemia successfully.
A lot of studies have proved the relationship between HUA and cardiovascular complications such as primary hypertension, left ventricular hypertrophy (LVH), heart failure and myocardial infarction. HUA could lead to ED, inflammation and proliferation of vascular smooth muscle cell by inducing intracellular oxidative stress, activating RAAS and decreasing nitric oxide (NO) generation [13]. The composition and release of a large amount of inflammatory factors and the blockage of vessel NO generation could deteriorate ED, while dysfunction of coronary vessels could induce coronary circulation disorders, both of which could ultimately lead to myocardial ischemia and angina [14-17]. Apart from myocardial ischemia, aggregation of SUA and the release of oxygen radicals when it's being generated also increased the mortality of congestive heart failure (CHF) patients $[18,19]$. The relationship between hyperuricemia and cardiovascular complications in general 
Table 3. Correlation between SUA and PCIPA

\begin{tabular}{lll}
\hline & PCIPA & \\
\cline { 2 - 3 } & $\mathrm{r}$ & $\mathrm{p}$ \\
\hline $\mathrm{SUA}, \mu \mathrm{mol} / \mathrm{l}$ & 0.5465 & 0.0000 \\
\hline
\end{tabular}

Table 4. Correlation between SUA and hs-CRP, IL-6

\begin{tabular}{llll}
\hline & & hs-CRP, ng/ml & IL-6, pg/ml \\
\hline SUA, $\mu \mathrm{mol} / \mathrm{l}$ & $\mathrm{r}$ & 0.4332 & 0.4666 \\
& $\mathrm{p}$ & 0.0075 & 0.0019 \\
\hline
\end{tabular}

Table 5. Correlation between PCIPA and hs-CRP, IL-6

\begin{tabular}{llll}
\hline & & hs-CRP, ng/ml & IL-6, pg/ml \\
\hline PCIPA, \% & $\mathrm{r}$ & 0.2982 & 0.3642 \\
& $\mathrm{p}$ & 0.0094 & 0.0013 \\
\hline
\end{tabular}

population has been widely recognized. The SUA level in CKD patients was substantially higher due to UA excretion disorders. Cardiac lesions were the major complication and cause of death in CKD patients, which resulted in 50\% mortality of the end-stage renal disease (ESRD) patients. 75\% patients had had cardiovascular lesions before receiving renal replacement therapy. Even in early-stage $\mathrm{CKD}$, the occurrence of cardiac lesions like LVH increased evidently. However, researches focusing on the relation between cardiovascular complications and hyperuricemia in CKD patients are rare. Ryota Yoshitomi [20] found a significant correlation between hyperuricemia and ventricular hypertrophy in women, but there was no significant correlation in men. Researches focusing on cardiovascular complications in early-stage CKD are even scarcer.

According to our results, there were obvious cardiac lesions in Group C, including inflammatory cell infiltration, congestion and fibrillation of interstitium, fibroblasts in small vessel walls and the increase of the amount of myocardial collagen I. Univariate research has proved a positive correlation between UA and PCIPA, which suggested that UA was significantly involved in cardiac lesions in early-stage CKD.

Even in CKD patients with only slight renal dysfunction, micro-inflammation is common all over body [21]. It is believed that the severity of inflammation is closely related to the occurrence and mortality of cardiovascular events [22].
Menon found that a high CRP level was an independent risk factor predicting the death of 3, 4 stages CKD and CVD patients [23]. hs-CRP is an acute phase protein synthesized by liver when the body is faced with inflammatory stimuli such as tissue injury, and is also one of the most sensitive indicators of cardiosvascular events. UA, an inflammation promotion factor, has been found to be related to many inflammation indicators including WBC, IL, INF- $\alpha$ and CRP. We found that the level of sera hs-CRP and IL-6 in Group $\mathrm{C}$ was significantly higher, indicating a more severe microinflammation in this group. In addition, hs-CRP and IL-6 levels were closely related to SUA, indicating that UA might participate in the formation of micro-inflammation in early $\mathrm{CKD}$, which is in accordance with many researches worldwide. We found that sera hs-CRP and IL-6 levels were in positive correlation to PCIPA, which showed HUA participated in the early cardiac lesions in CKD possibly by inducing micro-inflammation. Qin found that, even after removing other possible confounding factors, SUA was still positively related to CRP, suggesting that UA was related to the micro-inflammation of the system and could regulate the progress of chronic inflammation [24]. Moreover, studies have shown that UA could activate p38 MAPK, transcription factor NF- $\kappa \mathrm{B}$ and AP-1 in smooth muscle cells, which could facilitate the composition of monocyte chemotactic protein-1 (MCP-1) and increase the amount of cytokines, which played an important role in cardiovascular diseases and atherosclerosis [25-27]. It suggested that UA might participate in CVD by inducing inflammation, which was consistent with the findings of our experiment.

Allopurinol is a common competitive inhibitor of xanthine oxidase, which could reduce the generation of UA. Researches have also found its cardiovascular protective effects. A retrospective controlled study showed that only high-dose, long-term allopurinol could reduce the hospitalization rate and mortality of CHF caused by prolonged high concentration of urate [28]. A heart failure (HF) rat model proved that long-term treatment of allopurinol could improve cardiac hemodynamics, reduce myocardial hypertrophy and collagen deposition, reduce left ventricular dilation and ultimately improve cardiac systolic and diastolic functions [29]. However, the studies about the CVD protective effects of allopurinol were mainly focused on non-CKD patients. In this study, we found that after allopurinol treatment, SUA in Group D decreased significantly, and the severity of cardiac lesion and PCIPA was also significantly lower. Also, SUA was closely correlated to PCIPA, which suggested that allopurinol could treat CVD in CKD patients by lowering the SUA level. In our experiment, hs-CRP and IL-6 levels de- 
creased significantly after allopurinol interference. Additionally, PCIPA was in positive correlation to hs-CRP and IL-6, which indicated that allopurinol could improve cardiac lesions in CKD patients with hyperuricemia by reducing the severity of micro-inflammation. Lately, Goicoechea [30] found that compared to the control group, hs-CRP in CKD patients could be substantially lowered after treated with allopurinol for a year and micro-inflammation was largely reduced. Baldwin [31] found that after lowering UA in rats with allopurinol, endocrine disorders of adipose tissues, which could induce inflammation could be set right by reducing MCP-1 and increasing the synthesis of adiponectin.
To sum up, we established an early-stage CKD animal model with hyperuricemia by right nephrectomy and gavaging them with potassium oxonate, and we found out that UA could lead to cardiac lesions in early-stage CKD. UA might participate in the formation of micro-inflammation in early-stage CKD, which could lead to cardiac lesions. Allopurinol may significantly reduce cardiac lesions in CKD with hyperuricemia by both lowering the SUA level and reducing the severity of micro-inflammation in the body. Therefore, reducing the level of UA and the severity of micro-inflammation in early-stage CKD patients could improve cardiac lesions and therefore improve the quality of life of CKD patients greatly.

\section{References}

1 Gertler MM, Garn SM, Levine SA: Serum uric acid in relation to age and physique in health and in coronary heart disease. Ann Intern Med 1951;34:1421-1431.

2 Corry DB, Eslami P, Yamamoto K, et al: Uric acid stimulates vascular smooth muscle cell proliferation and oxidative stress via the vascular renin-angiotensin system. J Hypertens 2008;26:269-275.

- 3 Khosla UM, Zharikov S, Finch JL, et al: Hyperuricemia induces endothelial dysfunction. Kidney Int 2005;67:1739-1742.

4 Jossa F, Farinaro E, Panico S, et al: Serum uric acid and hypertension: the Olivetti heart study. J Hum Hypertens 1994;8:677-681.

$\checkmark 5$ Freedman DS, Williamson DF, Gunter EW, et al: Relation of serum uric acid to mortality and ischemic heart disease: the NHANES I epidemiologic follow-up study. Am J Epidemiol 1995;141:637-644.

6 Liese AD, Hense HW, Lowel H, et al: Association of serum uric acid with all-cause and cardiovascular disease mortality and incident myocardial infarction in the MONICA Augsburg cohort: World Health Organization monitoring trends and determinants in cardiovascular diseases. Epidemiology 1999;10:391-397.

7 Niskanen LK, Laaksonen DE, Nyyssonen K, et al: Uric acid level as a risk factor for cardiovascular and all-cause mortality in middleaged men: a prospective cohort study. Arch Intern Med 2004;164:1546-1551.

8 Foley RN, Parfrey PS, Sarnak MJ: Clinical epidemiology of cardiovascular disease in chronic renal disease. Am J Kidney Dis 1998; 32:S112-S119.

9 Ohno I: Relationship between hyperuricemia and chronic kidney disease. Nucleosides $\mathrm{Nu}$ cleotides Nucleic Acids 2011;30:1039-1044.

10 Wang Y, Bao X: Effects of uric acid on endothelial dysfunction in early chronic kidney disease and its mechanisms. Eur J Med Res 2013;18:26.

11 Szwejkowski BR, Gandy SJ, Rekhraj S, et al: Allopurinol reduces left ventricular mass in patients with type 2 diabetes and left ventricular hypertrophy. J Am Coll Cardiol 2013;62: 2284-2293.

12 Terawaki H, Nakayama M, Miyazawa E, et al: Effect of allopurinol on cardiovascular incidence among hypertensive nephropathy patients: the Gonryo study. Clin Exp Nephrol 2013; 17:549-553.

13 Mazzali M, Kanbay M, Segal MS, et al: Uric acid and hypertension: cause or effect? Curr Rheumatol Rep 2010;12:108-117.

14 Suwaidi JA, Hamasaki S, Higano ST, et al: Long-term follow-up of patients with mild coronary artery disease and endothelial dysfunction. Circulation 2000;101:948-954.

15 Halcox JP, Schenke WH, Zalos G, et al: Prognostic value of coronary vascular endothelial dysfunction. Circulation 2002;106:653-658.

16 Hasdai D, Gibbons RJ, Holmes DR, et al: Coronary endothelial dysfunction in humans is associated with myocardial perfusion defects. Circulation 1997;96:3390-3395.

17 Zeiher AM, Krause T, Schachinger V, et al: Impaired endothelium-dependent vasodilation of coronary resistance vessels is associated with exercise-induced ischemia. Circulation 1995;91:2345-2352.

18 Thanassoulis G, Brophy JM, Richard H, et al: Gout, allopurinol use, and heart failure outcomes. Arch Intern Med 2010;170:13581364.

19 Belch JJ, Bridges AB, Scott N, et al: Oxygen free radicals and congestive heart failure. $\mathrm{Br}$ Heart J 1991;65:245-248.

20 Yoshitomi R, Fukui A, Nakayama M, et al: Sex differences in the association between serum uric acid levels and cardiac hypertrophy in patients with chronic kidney disease. Hypertens Res 2014;37:246-252.

21 Kundhal K, Lok CE: Clinical epidemiology of cardiovascular disease in chronic kidney disease. Nephron 2005;101:47-52.

22 Arici M, Walls J: End-stage renal disease, atherosclerosis, and cardiovascular mortality: is
C-reactive protein the missing link? Kidney Int 2001;59:407-414.

23 Menon V, Greene T: C-reactive protein and albumin as predictors of all-cause and cardiovascular mortality in chronic kidney disease. Kidney Int 2005;68:766-772.

$24 \mathrm{Gu}$ L, Okada Y, Clinton SK, et al: Absence of monocyte chemoattractant protein-1 reduces atherosclerosis in low density lipoprotein receptor-deficient mice. Mol Cell 1998;2:275-281.

25 Kanellis J, Watanabe S, Li JH, et al: Uric acid stimulates MCP-1 production in vascular smooth muscle cells via mitogen-activated protein kinase and cyclooxygenase- 2 . Hypertension 2003;41:1287-1293.

26 Li Q, Yang Z, Lu B, et al: Serum uric acid level and its association with metabolic syndrome and carotid atherosclerosis in patients with type 2 diabetes. Cardiovasc Diabetol 2011;10:72.

27 Kang DH, Park SK, Lee IK, et al: Uric acidinduced C-reactive protein expression: implication on cell proliferation and nitric oxide production of human vascular cells. J Am Soc Nephrol 2005;16:3553-3562.

28 Struthers AD, Donnan PT, Lindsay P, et al: Effect of allopurinol on mortality and hospitalisations in chronic heart failure: a retrospective cohort study. Heart 2002;87:229-234.

29 Mellin V, Isabelle M, Oudot A, et al: Transient reduction in myocardial free oxygen radical levels is involved in the improved cardiac function and structure after long-term allopurinol treatment initiated in established chronic heart failure. Eur Heart J 2005;26:1544-1550.

30 Goicoechea M, de Vinuesa SG, Verdalles U, et al: Effect of allopurinol in chronic kidney disease progression and cardiovascular risk. Clin J Am Soc Nephrol 2010;5:1388-1393.

31 Baldwin W, McRae S, Marek G, Wymer D, et al: Hyperuricemia as a mediator of the proinflammatory endocrine imbalance in the adipose tissue in a murine model of the metabolic syndrome. Diabetes 2011;60:1258-1269. 